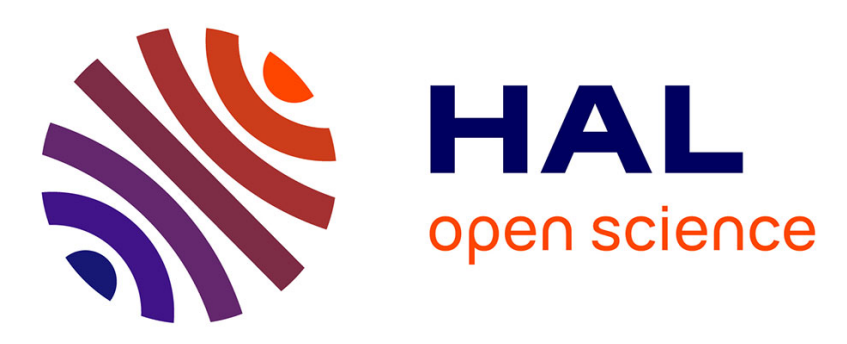

\title{
Automatic cardiac ventricle segmentation in MR images: a validation study
}

Damien Grosgeorge, Caroline Petitjean, Jérôme Caudron, Jeannette Fares, Jean-Nicolas Dacher

\section{- To cite this version:}

Damien Grosgeorge, Caroline Petitjean, Jérôme Caudron, Jeannette Fares, Jean-Nicolas Dacher. Automatic cardiac ventricle segmentation in MR images: a validation study. International Journal of Computer Assisted Radiology and Surgery, 2010, pp.1. 10.1007/s11548-010-0532-6 . hal-00524989

\section{HAL Id: hal-00524989 \\ https://hal.science/hal-00524989}

Submitted on 11 Oct 2010

HAL is a multi-disciplinary open access archive for the deposit and dissemination of scientific research documents, whether they are published or not. The documents may come from teaching and research institutions in France or abroad, or from public or private research centers.
L'archive ouverte pluridisciplinaire HAL, est destinée au dépôt et à la diffusion de documents scientifiques de niveau recherche, publiés ou non, émanant des établissements d'enseignement et de recherche français ou étrangers, des laboratoires publics ou privés. 


\title{
Automatic cardiac ventricle segmentation in MR images: a validation study
}

\author{
Damien Grosgeorge · Caroline Petitjean · \\ Jérôme Caudron • Jeannette Fares . \\ Jean-Nicolas Dacher
}

Received: date / Accepted: date

\begin{abstract}
Purpose Segmenting the cardiac ventricles in magnetic resonance (MR) images is required for cardiac function assessment. Numerous segmentation methods have been developed and applied to MR ventriculography. Quantitative validation of these segmentation methods with ground truth is needed prior to clinical use, but requires manual delineation of hundreds of images. We applied a well-established method to this problem and rigorously validated the results.

Methods An automatic method based on active contours without edges was used for left and the right ventricle cavity segmentation. A large database of $1920 \mathrm{MR}$ images obtained from 59 patients who gave informed consent was evaluated. Two standard metrics were used for quantitative error measurement.

Results Segmentation results are comparable to previously reported values in the literature. Since different points in the cardiac cycle and different slice levels were used in this study, a detailed error analysis is possible. Better performance was obtained at end diastole than at end systole, and on mid-ventricular slices than apical slices. Localization of segmentation errors were highlighted through a study of their spatial distribution.

Conclusions Ventricular segmentation based on region-driven active contours provided satisfactory results in MRI, without the use of a priori knowledge. The study of error distribution allows identification of potential improvements in algorithm performance.
\end{abstract}

Keywords Cardiac magnetic resonance imaging (CMRI) - Image segmentation · Ventricle segmentation · Validation · Active contours

D. Grosgeorge - C. Petitjean ( $\bowtie)$

Université de Rouen, LITIS EA 4108

BP 12, 76801 Saint-Etienne-du-Rouvray, France

Tel: +33232955215

Fax: + 33232955022

E-mail: caroline.petitjean@univ-rouen.fr

J. Caudron - J. Fares - J.-N. Dacher

University Hospital of Rouen, Department of Radiology

76031 Rouen, France 


\section{Introduction}

To study the cardiac function, MRI is a modality of choice that allows to obtain accurate anatomical and functional information [1]. The computation of clinical parameters to assess the cardiac function requires to segment the cardiac ventricles, as shown in Figure 1, where the left (LV) and right (RV) ventricles are identified. As the heart is a moving organ, images are acquired throughout the whole cardiac cycle, but two precise instants are of particular interest for the clinician: the time of maximum filling, when the heart is the most dilated (end diastole, ED) and the time of greatest contraction (end systole, ES). Although some relatively efficacious methods are commercially available for segmenting the LV, such as MASS (Medis, Leiden, The Netherlands) [2] and Argus (Siemens Medical Systems, Germany) [3], the segmentation of ED and ES images of the RV is currently performed manually in clinical routine. This long and tedious task, prone to intra and inter-expert variability, requires about 20 minutes per ventricle by a clinician. The great need for automated methods has led to the development of a wide variety of segmentation methods [4], among which thresholding [5], pixel classification [6-8], deformable models. This latter family of methods have been greatly used thanks to their flexibility, especially for this application [9-12], either on the form of $2 \mathrm{D}$ active contours or $3 \mathrm{D}$ deformable surfaces, which are more computationnally expensive $[13,14]$. Shape prior information can also be used to guide the segmentation process, under the form of a statistical model, in a variational framework [15], by using active shape and appearance models [16-20] or via an atlas, using registration-based segmentation $[21,22]$. Note that the temporal dimension of cardiac data can be taken into account to improve the segmentation process [13,23].

\subsection{The LV and RV segmentation challenge}

The challenges faced by all segmentation methods in cardiac MRI are: (i) fuzziness of the cavity borders due to blood flow, acquisition artefacts, and partial volume effect especially for apical slices [24], (ii) the presence of papillary muscles in the LV pool and trabeculations (wall irregularities) in the RV, which have the same grey level as the

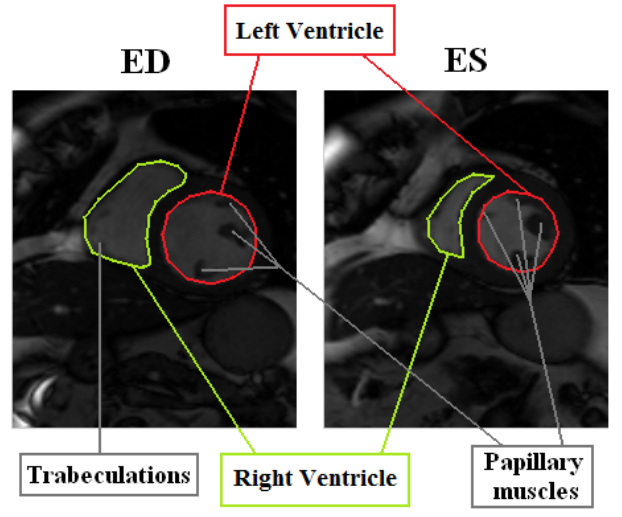

Fig. $1 \mathrm{LV}$, RV, papillary muscles and trabeculations on ED and ES images 
surrounding myocardium and yet must not be taken into account during segmentation, as shown on Figure 1, (iii) the complex crescent shape of the RV, which varies according to the imaging slice level (Figure 2). For this last reason, and because the RV function is less vital than the LV's, most research effort has focused on the LV, leaving the problem of RV segmentation wide open.

1.2 Choice of a segmentation methodology

To face these issues, deformable models have appeared as one of the most efficient approaches. Their principle is to iteratively deform an initial contour until it reaches the object frontiers to be detected, i.e. the LV and RV cavities. The deformation of the contour is driven by the minimization of an energy functional, that is designed to reach a minimum on the ventricle boundaries. Classically, the energy functional comprises two terms: a data-driven term that provides information about object frontiers and a regularization term that controls the smoothness of the curve. Initially edge-based and thus sensitive to noise, the data-driven term can be chosen to be region-based, such as in the well-known active contours without edges (ACWE) [25]. Region-based energy terms in a variational approach have been widely used in the literature of cardiac MR image segmentation $[26,12,11]$, since segmentation can sorely rely on the ventricle borders only. In curve evolution, the level set framework allows for automatic topological change, i.e. splitting and merging of the contour [27]. This enables multiple object segmentation, an interesting property to detect both ventricle cavities.

The ACWE model is thus a segmentation method that is computationally efficient, does not require user interaction, nor heavy postprocessing steps, nor the learning of a priori shape. Furthermore, thanks to the design of a region-based energy functional, the ACWE model can detect objects whose boundaries are not necessarily defined by the image gradient. This has lead us to choose this well-tried, preliminary approach for our segmentation problem.

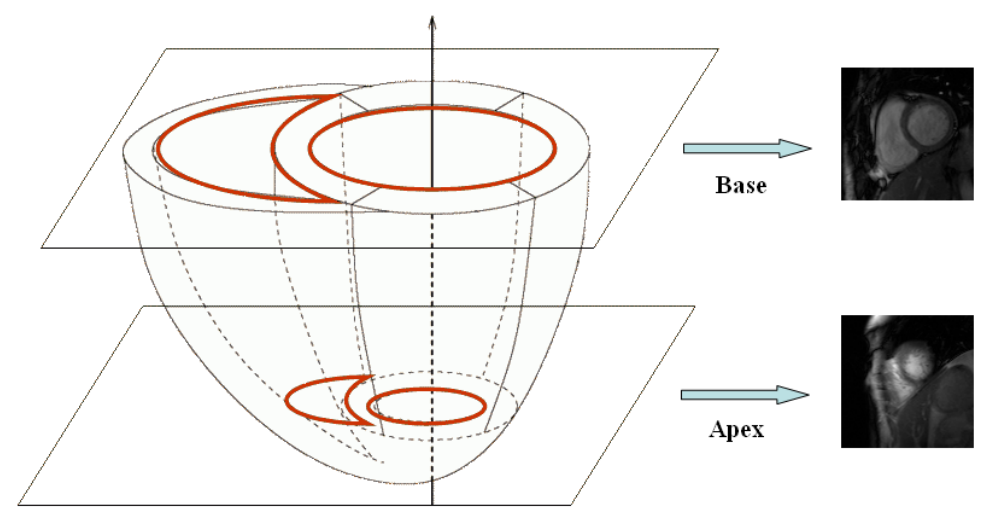

Fig. 2 Schematic representation of ventricle volumes and associated MR images obtained on basal and apical imaging slices 


\subsection{Validation issues}

Validation of the segmentation algorithm against ground truth, i.e. manual delineation, is of great importance. As manual segmentation is time-consuming, validation is often restricted to a few dozens or hundreds of images in the literature, obtained on a few patients. Additionally, results are often limited to mid-ventricular or basal slices, and at ED, where the heart presents the most regular and largest shapes, or sometimes provided on healthy volunteers, whereas images from pathological subjects are more prone to noise and artefacts, and thus, more difficult to segment. For this work, we propose the validation of our method over 1920 images, covering all slice levels, acquired on patients presenting different pathologies. We also suggest to sudy the spatial distribution of segmentation errors - which ventricle is easier to segment, which slice level, and at which instant of the cardiac cycle - so as to gain some insight on the segmentation difficulties and to identify where room for improvement is left. To the best of our knowledge, no such extensive segmentation tests nor study of the error distribution has been realized on these images.

In the remaining of the paper, we present the chosen segmentation method, based on active contours without edges, in Section 2. Validation and results are presented in Section 3 and conclusion and perspectives for this work are drawn in Section 4.

\section{Cardiac image segmentation method}

\subsection{Method basic principle}

The principle of our segmentation method is to define a single initial contour on our cardiac image, that evolves according to an evolution equation. In the level set framework, the contour automatically splits into several different regions, among which the ventricle cavities. The ventricle pools are identified as being the two largest connected components [28]. Residual components inside the cavities (if any) are removed. The $\mathrm{LV}$ and RV are labeled according to the position of their center of gravity (the RV is to the left of the LV). The different steps of our method are illustrated in Figure 3. In the following, the theoretical background and implementation details regarding the ACWE approach are provided.

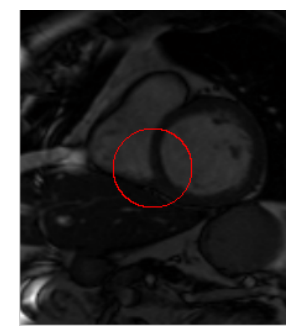

(a) Initial contour

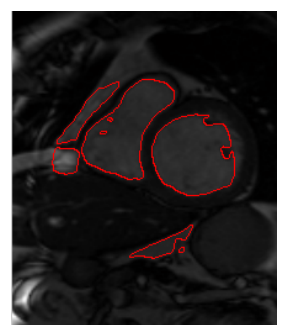

(b) After ACWE convergence

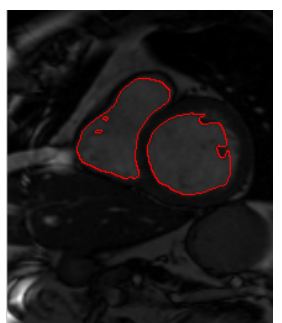

(c) After selection of the 2 largest components

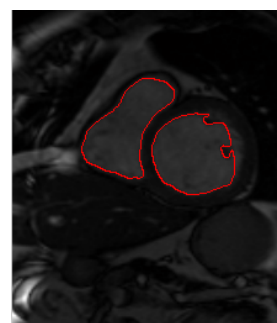

(d) After removal of inside contours

Fig. 3 The different steps of our method and corresponding evolution of the deformable contour 


\subsection{Theoretical background of the ACWE model}

The aim of the ACWE approach is to separate the image into regions based on their mean intensities. The image is considered as being composed of two regions of roughly uniform intensity [25]: one region is the inside of the contour (the ventricle cavities or foreground) of mean intensity $c_{1}$, and the other one, the outside (the rest of the image or background), of mean intensity $c_{2}$. The foreground and background distributions are assumed to be gaussian [26,29].

Let us denote by $C$ the deformable contour, $U$ the image, where $U(\mathbf{x})$ represents the pixel value at location $\mathbf{x}=(x, y)$. The region-based energy term is given by the following equation:

$$
E_{C V}(C)=\int_{\omega}\left|U(\mathbf{x})-c_{1}\right|^{2} d \mathbf{x}-\int_{\Omega \backslash \omega}\left|U(\mathbf{x})-c_{2}\right|^{2} d \mathbf{x}
$$

where $\Omega \in \Re^{2}$ represents the image domain and $\omega$ the domain inside the contour. This energy term is regularized by the contour curvature $\kappa$, a well-known regularizer [30], and the total energy $E(C)$ of the contour can thus be defined as:

$$
E(C)=E_{C V}(C)+\mu \kappa
$$

where $\mu$ is a user-defined weighting parameter. This energy functional is minimized by implementing the Euler-Lagrange equations in a partial differential equation (PDE), that allows to obtain the contour evolution equation. The minimization is performed via gradient descent, a method that can get trapped in local minima, when the contour is initialized too far from the boundaries to be reached [31-33].

The level set framework consists in considering the contour $C$ as the zero level of a two-dimensional function $\psi: C=\{\mathbf{x} \in \Omega: \psi(\mathbf{x})=0\}$ (Figure 4). The evolution of $\psi$ can then be written as [25]:

$$
\frac{\partial \psi}{\partial t}=\delta(\psi)\left(\mu \kappa-\left(U-c_{1}\right)^{2}+\left(U-c_{2}\right)^{2}\right)=0
$$

where $\delta(\cdot)$ denotes the Dirac function. Average intensities $c_{1}$ and $c_{2}$ are updated throughout the iterations as the contour evolves. The curvature $\kappa$ of the contour can be computed directly from $\psi$ using:

$$
\kappa=\operatorname{div}\left(\frac{\nabla \psi}{|\nabla \psi|}\right)
$$

2.3 Implementation details of the ACWE model

The segmentation algorithm applied to each cardiac MR image is composed of the following steps:

1. Contour initialization, as a circle centered on the image. The radius of the circle is equal to one eigth of the image width, an empirical value that has proven to be quite adequate in regard to the ventricle size. 


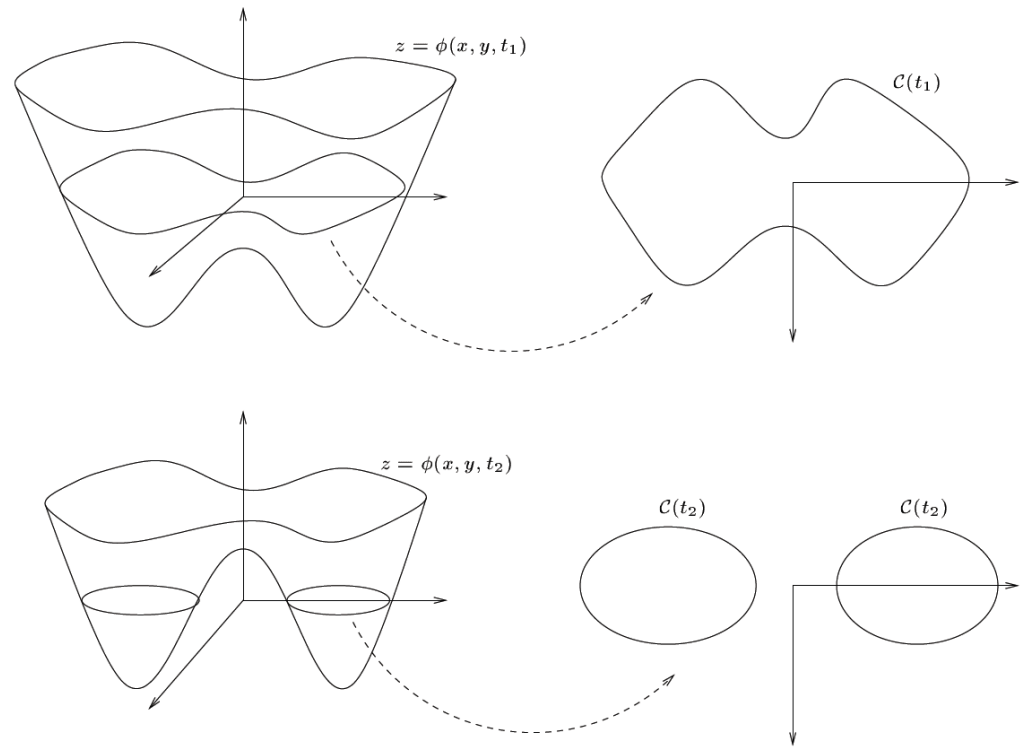

Fig. 4 The level set function $\psi$ at two different instants $t_{1}$ and $t_{2}$ and the contour $C$ as its zero level (from [34])

2. Initialization of the $\psi$ function with the signed distance map to the initial contour. 3. Updating of $\psi$ at each iteration, using the discrete version of Equation 3:

$$
\psi^{n+1}=\psi^{n}+\Delta t V
$$

where $\Delta t$ is the time step and $V$ is the discrete version of $\delta(\psi)\left(\mu \kappa-\left(U-c_{1}\right)^{2}+\right.$ $\left.\left(U-c_{2}\right)^{2}\right)$ in which $\mu$ is classically set to 0.3 . For further information regarding the formulation of $V$, the reader is refered to [25]. The time step value must be large enough not to slow the level set evolution, and small enough to ensure the stability of the numerical scheme [35]. The maximum value for the time step is given by the Courant-Friedrichs-Lewy (CFL) condition:

$$
\Delta t<\frac{\Delta x}{\max _{\Omega} V}
$$

where $\max _{\Omega} V$ is the maximum value of $V$ on the image domain and $\Delta x$ is the spatial step. This equation is usually enforced by introducing a CFL number $\alpha$, usually set to 0.5 [36]: $\Delta t=\alpha \frac{\Delta x}{\max \Omega_{\Omega} V}$. To guarantee the stability of numerical solutions, the CFL condition requires the boundary move no further than one spatial step after each time step [35]. The value of the time step is thus:

$$
\Delta t=\frac{0.5}{\max _{\Omega} V}
$$

We thus have an adaptative time step which verifies the CFL condition without adding an extra parameter to tune. The contour $C$ is obtained by identifying points $\mathbf{x}$ which are on the zero level of the level set function, i.e. such as $\psi(\mathbf{x})=0$. 
In order to keep the regularity of $\psi$ at each iteration, the $\psi$ function is reinitialized to the distance function through the resolution of the following PDE [37]:

$$
\frac{\partial \psi}{\partial t}=\operatorname{sign}\left(\psi_{0}\right)(1-|\nabla \psi|)
$$

where $\psi_{0}$ is the initial value of $\psi$. Note that methods without re-initialization of the level set function have been proposed [38], that decrease computation time. In order to speed up computation, $\psi$ is not updated on the whole image but only in the neighborhood of the contour points [39], i.e. on a narrow band. The width of this band should be equal to the frontier width of the object to be segmented as suggested by the literature [39]. According to MRI spatial resolution, the cavity borders have been assessed to be 1.2 pixels wide, the value chosen for the narrow band width.

After a certain number of iterations, the algorithm has converged: the level set does not evolve anymore. For sake of simplicity, we have chosen a fixed number of iterations (set to 120), a number large enough to guarantee that the ventricle borders have been reached.

We shall now introduce the database of cardiac images and study the performance of the segmentation method on this large dataset, as well as the spatial distribution of segmentation errors.

\section{Experimental results}

3.1 Cardiac image database and validation method

Our validation database is composed of 59 patients who gave informed consent, with identified pathologies, such as myocarditis, myocardial infarction and dilated cardiomyopathy. All MRI examinations are performed using a 1.5T MR scanner (Magnetom Symphony TIM; Siemens Healthcare, Erlangen, Germany). Images are 256 pixels x 216 pixels with a spatial resolution of $0.7 \mathrm{~mm}$ per pixel. For each patient, imaging slices cover the whole heart from the apex to the base of the heart, providing between 6 and 10 slice levels depending on the size of the heart, with a slice thickness of $7 \mathrm{~mm}$. The most basal short-axis slice is selected using the 4-chamber view, as the one crossing the tricuspid valve. The most apical slice is selected as the last slice showing white blood in the LV pool.

At each slice level, images at ED and at ES are selected, for a total of 1038 images of the LV and 882 for the RV. For each of them, the left ventricle and the right ventricle contours were manually drawn by a 4-year experienced cardiac radiologist of the University Hospital of Rouen, in clinical routine conditions. In order to study the influence of the slice level on segmentation errors, three groups of slices have been defined, following a standard nomenclature [40]: apical, mid-ventricular, and basal slice levels. For each patient, all imaging slices have been split into these three levels, and results on each group of slices have been obtained by averaging results obtained on individual slices in the corresponding group. The detailed number of images per group of slices is given in Table 1 .

Results obtained using our algorithm have been compared to manual segmentation through the computation of two standard and complementary error measurements: 
Table 1 Mean Dice Metric \pm standard deviation (DM) and false segmentation rates obtained for the LV and RV on different slice levels

\begin{tabular}{|c|c|c|c|c|c|}
\hline & & Nb. of images & Slice level & DM & False Seg. \\
\hline \multirow{6}{*}{ LV } & \multirow{3}{*}{$\mathrm{ED}$} & \multirow{3}{*}{569} & Base & $0.82 \pm 0.01$ & 0.07 \\
\hline & & & Mid & $0.75 \pm 0.01$ & 0.02 \\
\hline & & & Apex & $0.67 \pm 0.02$ & 0.14 \\
\hline & \multirow{3}{*}{ ES } & \multirow{3}{*}{469} & Base & $0.70 \pm 0.03$ & 0.16 \\
\hline & & & Mid & $0.58 \pm 0.00$ & 0.11 \\
\hline & & & Apex & $0.46 \pm 0.00$ & 0.35 \\
\hline \multirow{6}{*}{$\mathrm{RV}$} & \multirow{3}{*}{$\mathrm{ED}$} & \multirow{3}{*}{506} & Base & $0.80 \pm 0.01$ & 0.05 \\
\hline & & & Mid & $0.71 \pm 0.00$ & 0.13 \\
\hline & & & Apex & $0.46 \pm 0.00$ & 0.44 \\
\hline & \multirow{3}{*}{ ES } & \multirow{3}{*}{376} & Base & $0.59 \pm 0.01$ & 0.28 \\
\hline & & & Mid & $0.55 \pm 0.02$ & 0.29 \\
\hline & & & Apex & $0.25 \pm 0.04$ & 0.58 \\
\hline
\end{tabular}

- the Dice Metric [30], a measure of contour overlap, obtained by computing the intersection divided by the union of the two surfaces. Let us denote by $A_{a}$ (respectively $A_{m}$ ) the area enclosed by the automatic (respectively manual) segmentation, and $A_{\cap}=A_{m} \cap A_{a}$ the intersection of both areas. The Dice Metric $(D M)$ is defined as:

$$
D M\left(A_{a}, A_{m}\right)=\frac{2 A_{\cap}}{A_{a}+A_{m}}
$$

The DM varies from 0 (total mismatch) to 1 (perfect match).

- the Point to Curve (P2C) error, which is the mean perpendicular distance between both contours. Let us denote by $C_{a}$ (resp. $C_{m}$ ) the automatically (resp. manually) obtained contour. For each $C_{a}$ contour point $\mathbf{p}_{a}^{i}$, distances to all $C_{m}$ contour points $\mathbf{p}_{m}^{j}$ are computed. The minimum distance is retained and all minima are averaged into the P2C error, over all $N_{a}$ points of contour $C_{a}$ :

$$
P 2 C\left(C_{a}, C_{m}\right)=\frac{1}{N_{a}} \sum_{i=1}^{N_{a}} \min _{j} d\left(\mathbf{p}_{a}^{i}, \mathbf{p}_{m}^{j}\right)
$$

where $d(\cdot, \cdot)$ denotes Euclidean distance. A high value of P2C error indicates that contours do not match well.

\subsection{Segmentation results and analysis}

Some typical segmentation results obtained on the LV are presented in Figure 5 and on the RV on Figure 6. A false segmentation rate is computed, that indicates the number of missegmentation cases (when the automatic contour and the manual contour do not overlap at all) over the total number of segmentation results. Note that these missegmentation cases are not taken into account for the Dice Metric computation. The Dice Metric and false segmentation rates obtained on our validation database are summarized in Table 1 and mean P2C errors are presented in Table 2, along with segmentation errors collected from the literature. Note that many of the presented results were obtained using $3 \mathrm{D}$ segmentation algorithms, whereas our approach is a $2 \mathrm{D}$ segmentation method.

As one can see, segmentation results on cardiac images have been obtained on different number of patients, having diverse conditions, either only on ED, or restricted 
Table 2 Mean segmentation errors reported in the literature and for our approach. \%P: percentage of pathological subjects. ${ }^{a}$ No standard deviation provided. ${ }^{b} \mathrm{RMS}$ error. ${ }^{c}$ Leaveone-out protocol. ${ }^{*} 3 \mathrm{D}$ segmentation methodology.

\begin{tabular}{|c|c|c|c|c|c|c|}
\hline Authors & Nb. subj. & $\% \mathrm{P}$. & Phases & Slice nb. & $\mathrm{LV}(\mathrm{mm})$ & $\mathrm{RV}(\mathrm{mm})$ \\
\hline Lynch et al. [7] & 25 & - & ED, ES & $5-12$ & $0.69 \pm 0.88$ & - \\
\hline El Berbari et al. [9] & 13 & - & ED & 3 & $0.6 \pm 0.3$ & - \\
\hline Kaus et al. [15] & 121 & $100 \%$ & $\begin{array}{l}\text { ED } \\
\text { ES }\end{array}$ & $7-10$ & $\begin{array}{l}2.45 \pm 0.75 \\
2.84 \pm 1.05\end{array}$ & $\overline{-}$ \\
\hline Mitchell et al. [16] & 20 & $45 \%$ & ED & 3 Mid & $1.71 \pm 0.82^{b}$ & $2.46 \pm 1.39^{b}$ \\
\hline Mitchell et al. $[17]^{*}$ & $56^{c}$ & $32 \%$ & ED & $8-14$ & $2.75 \pm 0.86$ & - \\
\hline Van Assen et al. $[18]^{*}$ & 15 & $0 \%$ & ED & $10-12$ & $1.97 \pm 0.54$ & - \\
\hline Abi-Nahed et al. $[19]^{*}$ & 13 & - & ED & - & - & $1.1^{a}$ \\
\hline Lorenzo-V. et al. $[21]^{*}$ & 10 & $100 \%$ & $\begin{array}{l}\text { all } \\
\text { ED }\end{array}$ & $3 \mathrm{Mid}$ & $\begin{array}{l}2.21 \pm 2.22 \\
1.88 \pm 2.00\end{array}$ & $\begin{array}{l}2.89 \pm 2.56 \\
2.26 \pm 2.13\end{array}$ \\
\hline Lotjonen et al. $[22]^{*}$ & $25^{c}$ & $100 \%$ & ED & $4-5$ & $2.01 \pm 0.31$ & $2.37 \pm 0.5$ \\
\hline \multirow{2}{*}{ Our study } & \multirow{2}{*}{59} & \multirow{2}{*}{$100 \%$} & ES & $\begin{aligned} \text { Base } \\
\text { 6-10 Mid } \\
\text { Apex }\end{aligned}$ & $\begin{array}{l}2.45 \pm 1.57 \\
2.27 \pm 2.02 \\
5.00 \pm 2.12 \\
\end{array}$ & $\begin{array}{c}1.85 \pm 1.42 \\
2.38 \pm 1.89 \\
3.5 \pm 2.38\end{array}$ \\
\hline & & & ED & $\begin{array}{l}\text { Base } \\
\text { 6-10 Mid } \\
\text { Apex }\end{array}$ & $\begin{array}{l}2.33 \pm 1.78 \\
2.91 \pm 2.25 \\
3.52 \pm 2.05\end{array}$ & $\begin{array}{c}2.6 \pm 2.46 \\
2.27 \pm 2.02 \\
3.28 \pm 2.54\end{array}$ \\
\hline
\end{tabular}

to a few mid-ventricular slices, thus making it difficult to conclude on the efficiency or the superiority of one method over the others. Accurate segmentation results would be expected to reach intra and inter-observer variability of manual segmentation, that is, around $2 \mathrm{~mm}$ [18]. On the whole, P2C errors obtained with our approach compare favorably to this value, and are honorable compared to other results in the literature, but room for improvement exists, in particular on apical slices. We can also remark that papillary muscles are sometimes considered as part of the myocardium (when they are very close to the wall) by the algorithm, whereas the corresponding manual contouring excludes them from the segmentation, which contributes to increase the $\mathrm{P} 2 \mathrm{C}$ errors. Note that our approach is totally generic and is not based on a priori knowledge, whereas most of the approaches that are presented here use a statistical shape prior to help the segmentation.

Considering the DM and the false segmentation rate, results are better on the LV than on the RV, mainly due to the LV regular ring shape and lesser variability. Nevertheless, P2C errors tend to show that segmentation is more accurate on the RV. This is due to the residual presence of papillary muscles in LV automatic contouring, which considerably increases $\mathrm{P} 2 \mathrm{C}$ values, since manual contours are very smooth. Actually, the common current segmentation standards recommend to consider them as part as the ventricle pool and to contour only the wall, because manual segmentation is more reproductible in this case, than when papillary muscles are segmented as well. This provides smooth manual contouring, but the exact cavity volume computation should exclude the volume occupied by the papillary muscles. Indeed, in some automatic segmentation methods, papillary muscles are segmented as well, authors arguing that the radiologist should decide whether to incorporate them or not $[6,7]$.

Let us now study the spatio-temporal distribution of segmentation errors. Figures 7 and 8 show histograms of the distribution of the DM values over the whole image database, for the LV and RV, respectively. The distributions of P2C errors are shown in Figure 9 and 10. From these figures, one can observe that ED results are better than ES ones for any ventricle and any slice level. This can be explained by the fact that 
at ED, the ventricles are larger and less subject to noise. Results also depend upon slice levels: basal and mid-ventricular slice levels are simpler to segment than apical levels, where ventricle cavities are really small. Apical slices are more prone to partial volume effect, a consequence of which is a certain fuziness on the ventricle frontiers. Segmentation errors in apical images may originate from the initialization of the deformable contour, because of its fixed size that might not be optimal for these images. A solution would be to have an adaptative size for the initial contour, corresponding to the different slice levels [41].

Regarding computational cost, our algorithm is implemented in Java without any particular optimization and requires less than 7 seconds on a regular PC hardware to segment a single image, which represents less than 4 minutes for a patient, a time compatible with clinical practice.

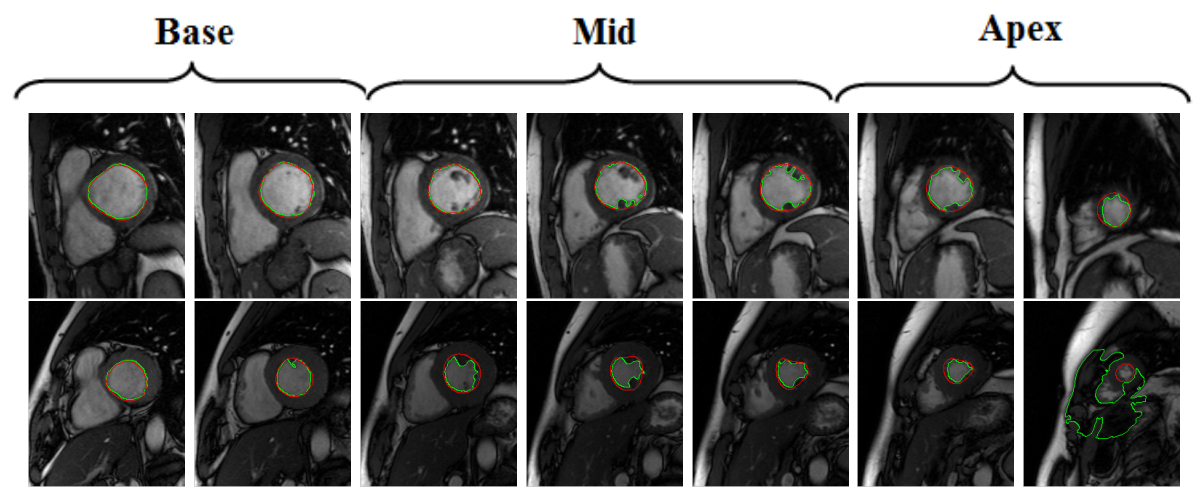

Fig. 5 Example of segmentation results on two different patients for the LV at ED (top) and ES (bottom). Red (resp. green) contours are obtained with manual (resp. automatic) segmentation. Note the missegmentation case for the last apical image.

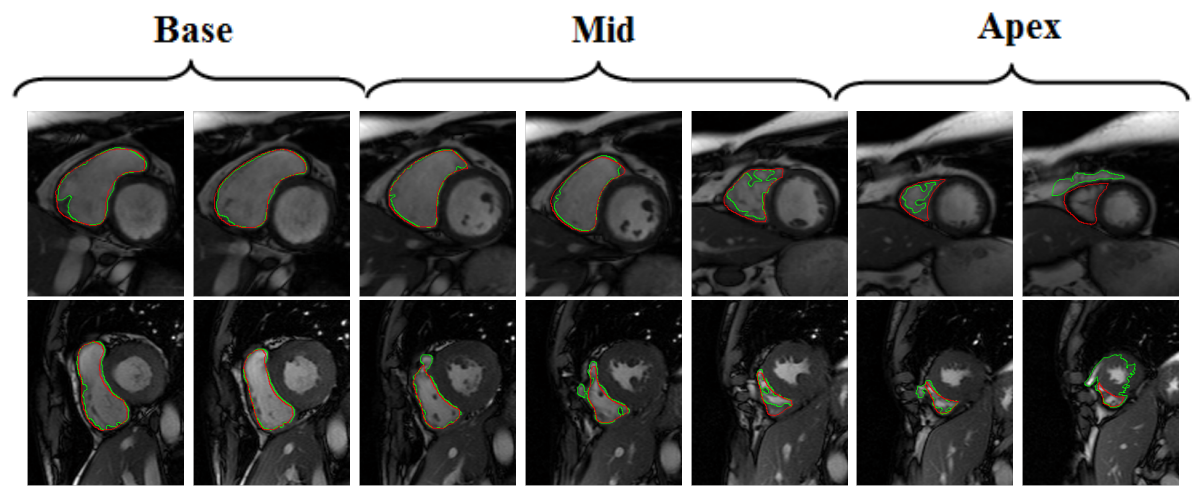

Fig. 6 Example of segmentation results on two different patients for the RV at ED (top) and ES (bottom). Red (resp. green) contours are obtained with manual (resp. automatic) segmentation. Note the missegmentation case for the last apical images. 


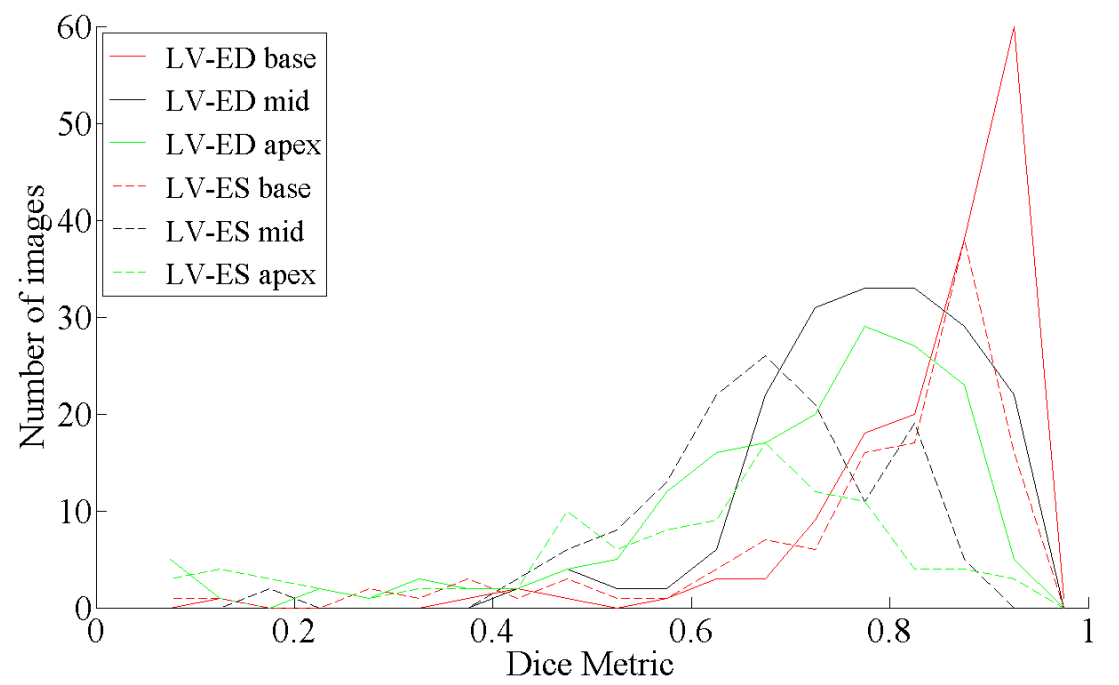

Fig. 7 Distribution of DM values for the LV over the whole image database

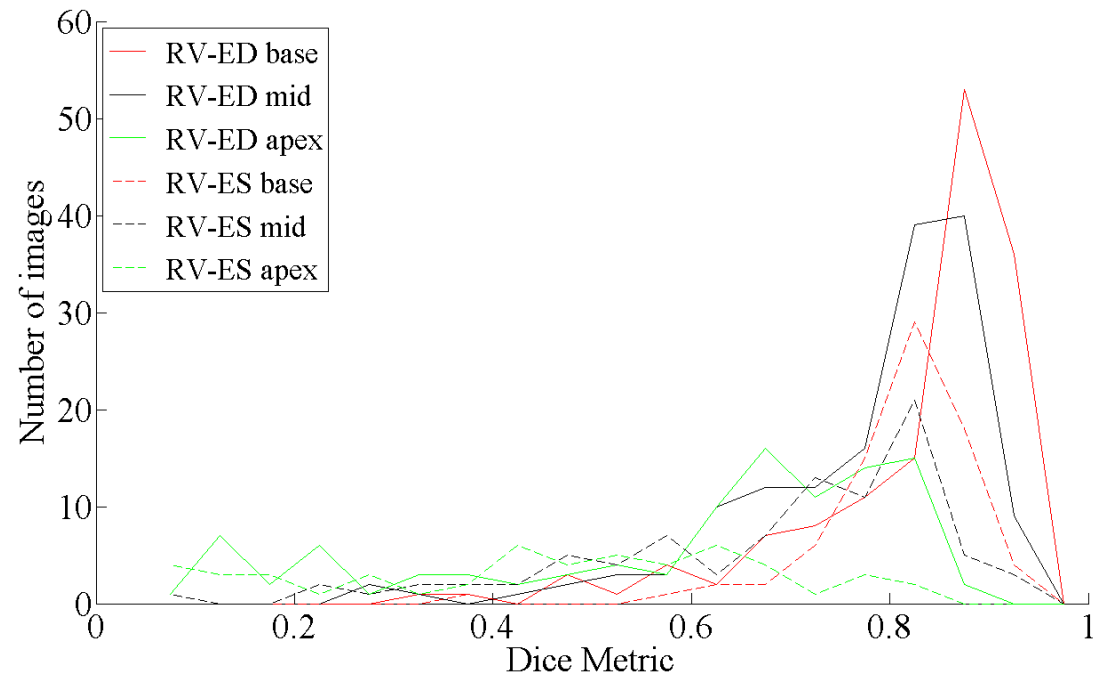

Fig. 8 Distribution of DM values for the RV over the whole image database 


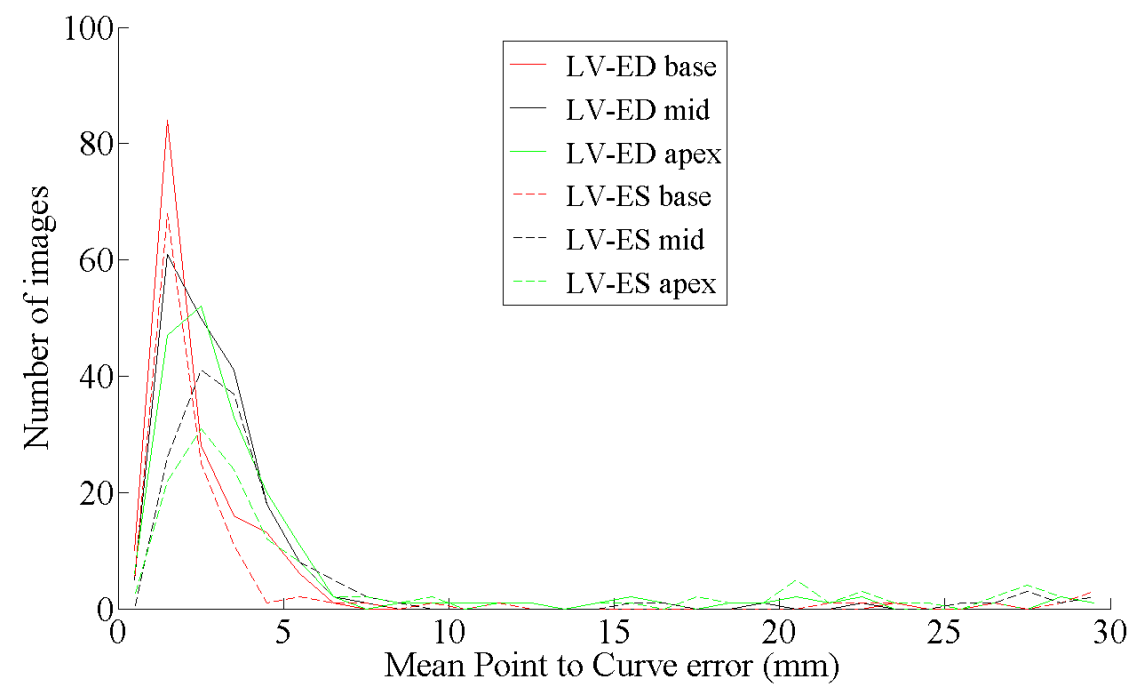

Fig. 9 Distribution of $\mathrm{P} 2 \mathrm{C}$ error values for the $\mathrm{LV}$ over the whole image database

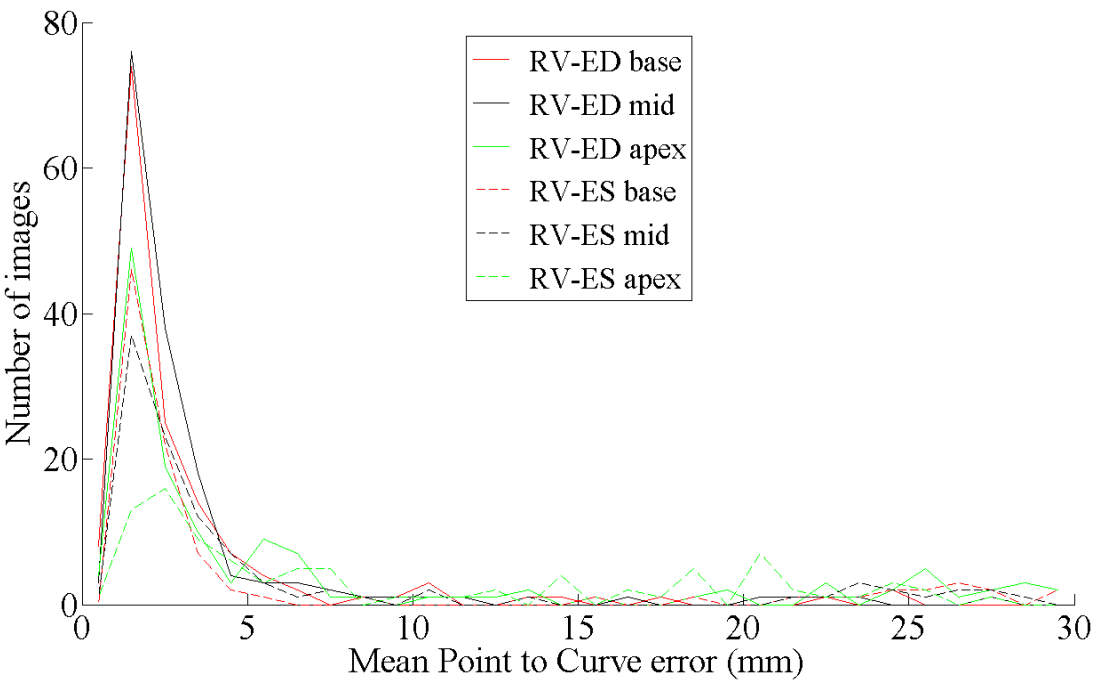

Fig. 10 Distribution of $\mathrm{P} 2 \mathrm{C}$ error values for the RV over the whole image database

\section{Conclusion and perspectives}

In this paper, we have been presenting the validation of an automatic method to perform the segmentation of both the left and right ventricles in cardiac MRI, by combining active contours without edges followed by a morphological step to smooth the resulting contour. This method allows for segmentation of both ventricle cavities. No user interaction is required since contour initialization is automatic. Validation has been 
performed on a very large database of 1920 images. An original study of segmentation errors show satisfying results on basal and mid-ventricular slices, compared to results provided by other segmentation methods in the litterature, and to manual segmentation accuracy. Nevertheless, segmentation errors obtained on apical slices and on the RV leave room for improvement. On some images, especially at the apex, myocardium borders are very fuzzy and ill-defined, and it is very difficult to rely on the image alone to perform segmentation. Perspectives for this work include (i) improving the accuracy of our segmentation approach by extending it to a 3D framework, which would allow the removal of residual papillary muscle contouring and improve apical segmentation results, (ii) applying the method to segment the epicardium of the heart, i.e. the outer border of the myocardium and comparing automatic results to manual ones, and (iii) the computation of clinical parameters: ventricule volume, mass and ejection fraction, and the correlation between manual tracing and our automatic method through linear regression and Bland-Altman analysis.

Acknowledgements The authors wish to thank Valentin Lefebvre, Sébastien Dubois and Que Nguyen for manually collecting the image data. They also would like to thank the anonymous reviewers whose insightful comments greatly helped to improve their paper. Damien Grosgeorge was supported by a grant from the Centre de Recherche et de Lutte Contre le Cancer (CRLCC) Henri Becquerel, Rouen, France.

\section{References}

1. J. Caudron, J. Fares, F. Bauer, and J.-N. Dacher. Left ventricular diastolic function assessment by cardiac MRI. RadioGraphics, in press (2011)

2. R. van der Geest, E. Jansen, V. Buller, and J. Reiber. Automated detection of left ventricular epi- and endocardial contours in short-axis MR images. In Computers in Cardiology, pages 33-36, Bethesda, MD, USA (1994)

3. T. O'Donnell, G. Funka-Lea, H. Tek, M.-P. Jolly, and M. Rasch. Comprehensive cardiovascular image analysis using $\mathrm{MR}$ and $\mathrm{CT}$ at Siemens Corporate Research. International Journal of Computer Vision, 70(2):165-178 (2006)

4. A.F. Frangi, W.J. Niessen, and M.A. Viergever. Three-dimensional modeling for functional analysis of cardiac images: A review. IEEE Trans. Med. Imaging, vol. 20, no. 1 (2001)

5. A. Goshtasby and D. Turner. Segmentation of cardiac cine MR images for extraction of right and left ventricular chambers. IEEE Transactions on Medical Imaging, 14(1):56-64 (1995)

6. A. Pednekar, U. Kurkure, R. Muthupillai, and S. Flamm. Automated left ventricular segmentation in cardiac MRI. IEEE Trans Biomed Eng, 53(7):1425-1428 (2006)

7. M. Lynch, O. Ghita, and P. Whelan. Automatic segmentation of the left ventricle cavity and myocardium in MRI data. Comput Biol Med, vol. 36, no. 4, pp. 389-407 (2006)

8. U. Kurkure, A. Pednekar, R. Muthupillai, S. Flamm, and I.A. Kakadiaris. Localization and Segmentation of Left Ventricle in Cardiac Cine-MR Images. IEEE Trans Biomed Eng, 56(5):1360-1370 (2009)

9. R. El Berbari, I. Bloch, A. Redheuil, E. Angelini, E. Mousseaux, F. Frouin, and A. Herment. An automated myocardial segmentation in cardiac MRI. in Conf Proc IEEE Eng Med Biol Soc., pp.4508-4511 (2007)

10. C. Xu, D. L. Pham, and J. L. Prince. Medical image segmentation using deformable models. In Handbook of Medical Imaging - Volume 2: Medical Image Processing and Analysis, pages 129-174. SPIE Press (2000)

11. N. Paragios, A variational approach for the segmentation of the left ventricle in cardiac image analysis. International Journal of Computer Vision, vol. 50, no. 3, pp. 345-362 (2002)

12. A. Chakraborty, L. Staib, and J.S. Duncan. Deformable boundary finding in medical images by integrating gradient and region information. IEEE Transactions on Medical Imaging, vol. 15, pp. 859-870 (1996) 
13. Y. Zhu, X. Papademetris, A.J. Sinusas, and J.S. Duncan. Segmentation of the Left Ventricle from Cardiac MR Images Using a Subject-Specific Dynamical Model. IEEE Transactions on Medical Imaging, 29(3):669-687 (2010)

14. J. Montagnat and H. Delingette. 4D deformable models with temporal constraints: application to 4D cardiac image segmentation. Medical Image Analysis, vol. 9, no. 1, pp. 87-100 (2005)

15. M. Kaus, J. von Berg, J. Weese, W. Niessen, and V. Pekar. Automated segmentation of the left ventricle in cardiac MRI. Medical Image Analysis, vol. 8, no. 3, pp. 245-254 (2004)

16. S. Mitchell, B. Lelieveldt, R. van der Geest, J. Bosch, J. Reiber, and M. Sonka. Multistage hybrid active appearance model matching: segmentation of left and right ventricles in cardiac MR images. IEEE Transactions on Medical Imaging, vol. 20, no. 5, pp. 415-423 (2001)

17. S. Mitchell, J. Bosch, B. Lelieveldt, R. van der Geest, J. Reiber, and M. Sonka. 3-D active appearance models: segmentation of cardiac $\mathrm{MR}$ and ultrasound images. IEEE Transactions on Medical Imaging, vol. 21, no. 9, pp. 1167-1178 (2002)

18. H.C. van Assen, M. Danilouchkine, A. Frangi, S. Ordas, J. Westenberg, J.H.C. Reiber, and B.P.F. Lelieveldt. SPASM: A 3D-ASM for segmentation of sparse and arbitrarily oriented cardiac MRI data. Medical Image Analysis, 10(2):286-303 (2006)

19. J. Abi-Nahed, M.-P. Jolly, and G.-Z. Yang. Robust active shape models: A robust, generic and simple automatic segmentation tool. in Proc. of Medical Image Computing and Computer-Assisted Intervention (MICCAI), number 2, pp. 1-8 (2006)

20. A. Andreopoulos and J.K. Tsotsos. Efficient and generalizable statistical models of shape and appearance for analysis of cardiac MRI. Medical Image Analysis, vol. 12, no. 3, pp. 335-357 (2008)

21. M. Lorenzo-Valdes, G. Sanchez-Ortiz, A. Elkington, R. Mohiaddin, and D. Rueckert. Segmentation of $4 \mathrm{D}$ cardiac MR images using a probabilistic atlas and the EM algorithm. Medical Image Analysis, 8(3):255-265 (2004)

22. J. Lötjönen, S. Kivistö, J. Koikkalainen, D. Smutek, and K. Lauerma. Statistical shape model of atria, ventricles and epicardium from short- and long-axis MR images. Medical Image Analysis, vol. 8, no. 3, pp. 371-386 (2004)

23. H. Zhang, A. Wahle, R.K. Johnson, T.D. Scholz, and M. Sonka. 4-D Cardiac MR Image Analysis: Left and Right Ventricular Morphology and Function. IEEE Transactions on Medical Imaging, 29(2):350-364 (2010)

24. C.B. Higgins and A. de Roos. MRI and CT of the cardiovascular system. Lippincott Williams \& Wilkins, (2006)

25. T.F. Chan and L.A. Vese. Active contours without edges. IEEE Transactions on Medical Imaging, 10(2):266-277 (2001)

26. C. Pluempitiwiriyawej, J. Moura, Y. Wu, and C. Ho. STACS: New active contour scheme for cardiac MR image segmentation. IEEE Transactions on Medical Imaging, vol. 24, no. 5, pp. 593-603 (2005)

27. S. Osher and J.A. Sethian. Fronts propagating with curvature-dependent speed: Algorithms based on HamiltonJacobi formulation. J. Comput. Phys., 79:12-49 (1988)

28. G. Kedenburg, C. Cocosco, U. Köthe, W. Niessen, E. Vonken, and M. Viergever. Automatic cardiac MRI myocardium segmentation using graphcut Proceedings of SPIE, number 6144 in Medical Imaging (2006)

29. X. Qian, H.D. Tagare, and Z. Tao. Segmentation of Rat Cardiac Ultrasound Images With Large dropout Regions. Proceedings of IEEE Computer Society Workshop on Mathematical Methods in Biomedical Image Analysis (MMBIA) (2006)

30. M. Lynch, O. Ghita, and P. Whelan. Segmentation of the left ventricle of the heart in $3 \mathrm{D}+\mathrm{t}$ MRI data using an optimised non-rigid temporal model. IEEE Transactions on Medical Imaging, 27(2):195-203 (2008)

31. G. Storvik. A Bayesian Approach to Dynamic Contours Through Stochastic Sampling and Simulated Annealing. IEEE Transactions on PAMI, 16(10):976-986, (1994)

32. H. Li, A. Yezzi. Local or Global Minima: Flexible Dual-Front Active Contours. IEEE Transactions on PAMI, 29(1):1-14, (2007)

33. G. Sundaramoorthi, A. Yezzi, A.C. Mennucci. Coarse-to-Fine Segmentation and Tracking Using Sobolev Active Contours. IEEE Transactions on PAMI, 30(5):851-864, (2008)

34. R. Keriven. Partial Differential Equations, Curves and Surface Evolutions and Scale-Spaces in Computer Vision. PhD Thesis, Ecole des Ponts ParisTech, Paris, France (1997)

35. Q. Xia, M.Y. Wang, S. Wang and S. Chen. Semi-Lagrange method for level-set-based structural topology and shape optimization. Structural and Multidisciplinary Optimization, vol. 31, no. 6 (2006) 
36. S. Osher and R.P. Fedkiw. Level set methods and dynamic implicit surfaces. Springer, (2003)

37. M. Sussman, E. Fatemi, P. Smereka, and S. Osher. An improved level set method for incompressible two-phase flows. Computers and Fluids, 27(5-6):663-680 (1997)

38. C. Li, C. Xu, C. Gui, and M.D. Fox. Level Set Evolution Without Re-initialization: A New Variational Formulation. IEEE International Conference on Computer Vision and Pattern Recognition (CVPR), San Diego, 1:430-436 (2005)

39. D. Adalsteinsson and J.A. Sethian. A fast level set method for propagating interfaces. Journal of Computational Physics, 118(2):269-277 (1995)

40. M.D. Cerqueira, N.J. Weissman NJ, V. Dilsizian, A.K. Jacobs, S. Kaul, W.K. Laskey, D.J. Pennell, J.A. Rumberger, T. Ryan, and M.S. Verani. Standardized myocardial segmentation and nomenclature for tomographic imaging of the heart. Circulation, 105(4):539-42 (2002)

41. Y. Zheng, A. Barbu, B. Georgescu, M. Scheuering, and D. Comaniciu. Four-Chamber Heart Modeling and Automatic Segmentation for 3-D Cardiac CT Volumes Using Marginal Space Learning and Steerable Features. IEEE Transactions on Medical Imaging, 27(11):1668$1681(2008)$ 